\title{
EXACT BOUNDS ON THE CLOSENESS BETWEEN THE STUDENT AND STANDARD NORMAL DISTRIBUTIONS
}

\author{
IOSIF PINELIS ${ }^{1}$
}

\begin{abstract}
Upper bounds on the Kolmogorov distance (and, equivalently in this case, on the total variation distance) between the Student distribution with $p$ degrees of freedom $\left(\mathrm{SD}_{p}\right)$ and the standard normal distribution are obtained. These bounds are in a certain sense best possible, and the corresponding relative errors are small even for moderate values of $p$. The same bounds hold on the closeness between $\mathrm{SD}_{p}$ and $\mathrm{SD}_{q}$ with $q>p$. Comparisons with known bounds are made.
\end{abstract}

Mathematics Subject Classification. 62E17, 60E15, 62E20, 62E15.

Received June 13, 2013. Revised May 19, 2014.

\section{SUMmaRY AND DISCUSSION}

The density and distribution functions of Student's distribution with $p$ degrees of freedom $\left(\mathrm{SD}_{p}\right)$ are given, respectively, by the formulas

$$
\begin{aligned}
f_{p}(x) & :=\frac{\Gamma\left(\frac{p+1}{2}\right)}{\sqrt{\pi p} \Gamma\left(\frac{p}{2}\right)}\left(1+\frac{x^{2}}{p}\right)^{-(p+1) / 2} \text { and } \\
F_{p}(x) & :=\int_{-\infty}^{x} f_{p}(u) \mathrm{d} u
\end{aligned}
$$

for all real $p>0$ and all real $x$. Let us extend definitions (1.1) and (1.2) by continuity to $p=\infty$, so that

$$
f_{\infty}=: \varphi \text { and } F_{\infty}=: \Phi
$$

are the density and distribution functions of the standard normal distribution (SND).

It is a textbook fact that the $\mathrm{SD}_{p}$ is close to the SND when $p$ is large, say in the sense that $f_{p}(x) \underset{p \rightarrow \infty}{\longrightarrow} f_{\infty}(x)$ for each real $x$. By Scheffé's theorem [8], this implies the convergence of the total variation distance

$$
d_{\mathrm{TV}}(p)=\frac{1}{2} \int_{-\infty}^{\infty}\left|f_{p}(x)-\varphi(x)\right| \mathrm{d} x
$$

to 0 as $p \rightarrow \infty$. In fact, the convergence of the $\mathrm{SD}_{p}$ to the $\mathrm{SND}$ is presented in [8] as the motivating case.

Keywords and phrases. Student's distribution, standard normal distribution, Kolmogorov distance, total variation distance, probability inequalities.

1 Department of Mathematical Sciences, Michigan Technological University, Houghton, 49931 Michigan, USA. ipinelis@mtu. edu 
Consider also the Kolmogorov distance

$$
d_{\mathrm{Ko}}(p):=\sup _{x \in \mathbb{R}}\left|F_{p}(x)-\Phi(x)\right|
$$

between the $\mathrm{SD}_{p}$ and $\mathrm{SND}$. It is clear that, for any two probability distributions, the Kolmogorov distance between them is no greater than twice the total variation distance, and hence the convergence of the latter distance to 0 implies that of the former.

However, in the present case one can say more. For any $p$ and $q$ in the interval $(0, \infty]$, let $d_{\text {Ko }}(p, q)$ and $d_{\mathrm{TV}}(p, q)$ denote, respectively, the Kolmogorov distance and the total variation distance between $\mathrm{SD}_{p}$ and $\mathrm{SD}_{q}$, so that $d_{\mathrm{Ko}}(p)=d_{\mathrm{Ko}}(p, \infty)$ and $d_{\mathrm{TV}}(p)=d_{\mathrm{TV}}(p, \infty)$.

\section{Proposition 1.1.}

(i) For all $p$ and $q$ such that $0<p<q \leq \infty$

$$
\frac{1}{2} d_{\mathrm{TV}}(p, q)=d_{\mathrm{Ko}}(p, q)=\max _{x \in(0, \infty)}\left(F_{q}(x)-F_{p}(x)\right) .
$$

(ii) Moreover, for each $p \in(0, \infty)$ the distance $d_{\mathrm{Ko}}(p, q)$ is strictly increasing in $q \in[p, \infty]$, and for each $q \in(0, \infty]$ the distance $d_{\mathrm{Ko}}(p, q)$ is strictly decreasing in $p \in(0, q]$. In particular,

$$
d_{\mathrm{Ko}}(p, q)<d_{\mathrm{Ko}}(p, \infty)=d_{\mathrm{Ko}}(p)
$$

for all $p$ and $q$ such that $0<p \leq q<\infty$, and $d_{\mathrm{Ko}}(p)$ is strictly decreasing in $p \in(0, \infty]$.

Statement (ii) holds as well with $d_{\mathrm{TV}}$ in place of $d_{\mathrm{Ko}}$.

This proposition and the other results stated in this section will be proved in Section 2.

The motivation for this study comes from the discussion in [4]. In turn, the paper [4] was motivated by developments of $[6]$.

Theorem 1.2. For any real $p \geq 4$

$$
\frac{1}{2} d_{\mathrm{TV}}(p)=d_{\mathrm{Ko}}(p)<C / p,
$$

where

$$
C:=\frac{1}{4} \sqrt{\frac{7+5 \sqrt{2}}{\pi e^{1+\sqrt{2}}}}=0.158 \ldots
$$

Moreover,

$$
\lim _{p \rightarrow \infty} p d_{\mathrm{Ko}}(p)=C,
$$

so that the constant $C$ is the best possible one in (1.6).

Corollary 1.3. For all $p$ and $q$ such that $4 \leq p<q \leq \infty$

$$
\frac{1}{2} d_{\mathrm{TV}}(p, q)=d_{\mathrm{Ko}}(p, q)<C / p .
$$

Graphs suggest that the bound $C / p$ is very close to $d_{\mathrm{Ko}}(p)$ for all $p \geq 4$, even in terms of the relative error (see [5], Figs. 1 and 2). Moreover, by (1.8), the relative error $\frac{C / p}{d_{\mathrm{Ko}}(p)}-1$ of the upper bound $C / p$ goes to 0 as $p \rightarrow \infty$. This convergence appears to be rather fast. E.g., for $p=12$, the relative and absolute errors of the bound $C / p$ are less than $1.5 \%$ and $2 \times 10^{-4}$. One may as well note that, if the distance $d_{\mathrm{Ko}}(p)$ is considered as a kind of "initial" error - of the approximation of the Student distribution by the SND, then the relative error $\frac{C / p}{d_{\mathrm{Ko}}(p)}-1$ is a relative error "of the second order", in the sense that it is the relative error of the estimate $C / p$ of the initial error $d_{\mathrm{Ko}}(p)$. Comparisons with results in $[1,2,7,9]$ can also be found in [5]. 


\section{Proofs}

Introduce

$$
r_{p, q}(x):=\frac{f_{p}(x)}{f_{q}(x)} \quad \text { and } \quad r_{p}(x):=r_{p, \infty}(x)=\frac{f_{p}(x)}{\varphi(x)}
$$

Lemma 2.1. For each pair $(p, q)$ such that $0<p<q \leq \infty$

(i) the ratio $r_{p, q}(x)$ decreases in $x \in[0,1]$ from $r_{p, q}(0)<1$, and then increases in $x \in[1, \infty)$ to $\infty$; therefore,

(ii) there is a unique point $x_{p, q} \in(0, \infty)$ (which is in fact greater than 1 ) such that

$$
\begin{aligned}
& f_{p}(x)<f_{q}(x) \text { for all } x \in\left[0, x_{p, q}\right), \\
& f_{p}\left(x_{p, q}\right)=f_{q}\left(x_{p, q}\right), \\
& f_{p}(x)>f_{q}(x) \text { for all } x \in\left(x_{p, q}, \infty\right),
\end{aligned}
$$

and hence

$$
d_{\mathrm{Ko}}(p, q)=F_{q}\left(x_{p, q}\right)-F_{p}\left(x_{p, q}\right) .
$$

Proof of Lemma 2.1. Take indeed any $p$ and $q$ such that $0<p<q \leq \infty$. A key observation here (borrowed from [3]) is that $r_{p, q}(x)$ decreases in $x \in[0,1]$ and increases in $x \in[1, \infty)$. Moreover, by Lemma 2.1 in [3], $f_{p}(0)$ increases in $p>0$ and hence $r_{p, q}(0)<1$. On the other hand, it is easy to see that $r_{p, q}(x) \rightarrow \infty$ as $x \rightarrow \infty$. This completes the proof of part (i) of Lemma 2.1, which in turn implies that there is a unique $x_{p, q} \in(0, \infty)$ such that $r_{p, q}(x)<1$ for $x \in\left[0, x_{p, q}\right), r_{p, q}\left(x_{p, q}\right)=1$, and $r_{p, q}(x)>1$ for $x \in\left(x_{p, q}, \infty\right)$ (at that necessarily $\left.x_{p, q}>1\right)$. In other words, one has the relations $(2.2)$. Since $\left(F_{q}-F_{p}\right)^{\prime}=f_{q}-f_{p}$, one now sees that $F_{q}(x)-F_{p}(x)$ increases in $x \in\left[0, x_{p, q}\right]$ from 0 to $F_{q}\left(x_{p, q}\right)-F_{p}\left(x_{p, q}\right)>0$, and then decreases in $x \in\left[x_{p, q}, \infty\right)$ to 0 . So, (2.3) follows by the symmetry of the Student and standard normal distributions. Thus, the lemma is completely proved.

Proof of Proposition 1.1. Take indeed any $p$ and $q$ such that $0<p<q \leq \infty$. By Lemma 2.1 and the symmetry of the $\mathrm{SD}_{p}$,

$$
d_{\mathrm{TV}}(p, q)=\int_{0}^{x_{p, q}}\left(f_{q}-f_{p}\right)+\int_{x_{p, q}}^{\infty}\left(f_{p}-f_{q}\right)=2 \int_{0}^{x_{p, q}}\left(f_{q}-f_{p}\right)=2\left(F_{q}\left(x_{p, q}\right)-F_{p}\left(x_{p, q}\right)\right)=2 d_{\mathrm{Ko}}(p, q),
$$

which proves part (i) of Proposition 1.1. Part (ii) of the proposition now follows by the second equality in (1.4) and the stochastic monotonicity result of [3], which implies that $F_{p}(x)$ is strictly increasing in $p \in(0, \infty]$ for each $x \in(0, \infty)$.

Proof of Theorem 1.2. This proof is based on a number of more or less technical statements in [5]. Indeed, the relations in (1.6) immediately follow by Theorem 1.3 and Lemma 2.6 in [5]. It remains to verify (1.8). First here, use l'Hospital's rule to find that for all real $x$

$$
\lim _{a \downarrow 0} \frac{f_{1 / a}(x)-f_{\infty}(x)}{a}=\lim _{a \downarrow 0} \frac{\partial f_{1 / a}(x)}{\partial a}=\lambda(x):=\frac{x^{4}-2 x^{2}-1}{4} \varphi(x) .
$$

Next, introduce

$$
c_{a}:=f_{1 / a}(0) \quad \text { and } \quad g_{a}(x)=f_{1 / a}(x) / c_{a}
$$

for all real $a \geq 0$, assuming the convention $1 / 0:=\infty$, so that $f_{1 / a}(x)=c_{a} g_{a}(x)$. Then for all real $a \geq 0$ and all real $x$

$$
\left|f_{1 / a}(x)-f_{\infty}(x)\right| \leq\left|c_{a}-c_{0}\right| g_{a}(x)+c_{0}\left|g_{a}(x)-g_{0}(x)\right| \leq\left|c_{a}-c_{0}\right|+\left|g_{a}(x)-g_{0}(x)\right|,
$$


since $g_{a}(x) \leq 1$ and $c_{0}=1 / \sqrt{2 \pi}<1$. By (2.4) and (2.5), the ratio $\frac{\left|c_{a}-c_{0}\right|}{a}$ is continuous in $a>0$ and converges to a finite limit $(\varphi(0) / 4)$ as $a \downarrow 0$, and hence is bounded in $a \in(0,1]$. Now note that

$$
\left|\frac{\partial g_{a}(x)}{\partial a}\right|=\left(1+a x^{2}\right)^{-(1+3 a) /(2 a)}|(D g)(a, x)| \leq|(D g)(a, x)|,
$$

where

$$
(D g)(a, x):=\frac{\left(1+a x^{2}\right) \ln \left(1+a x^{2}\right)-a(1+a) x^{2}}{2 a^{2}} .
$$

Using the Taylor expansion $\ln (1+u)=u-\theta u^{2} / 2$ for $u>0$ and some $\theta=\theta(u) \in(0,1)$, one sees that $(D g)(a, x)$ is a polynomial in $a, x, \theta$ and hence bounded in $(a, x) \in(0,1] \times\left[0, \tilde{x}_{0}\right]$ - note that, in accordance with the definition of $\tilde{x}_{a}$ in Theorem 1.3 in [5],

$$
\tilde{x}_{0}=\sqrt{1+\sqrt{2}} \in(0, \infty)
$$

hence, $\left|\frac{\partial g_{a}(x)}{\partial a}\right|$ is bounded in $(a, x) \in(0,1] \times\left[0, \tilde{x}_{0}\right]$ and, by the mean value theorem, so is $\frac{\left|g_{a}(x)-g_{0}(x)\right|}{a}$. Recalling also (2.6) and that the ratio $\frac{\left|c_{a}-c_{0}\right|}{a}$ is bounded in $a \in(0,1]$, one concludes that the ratio $\frac{\left|f_{1 / a}(x)-f_{\infty}(x)\right|}{a}$ is bounded in $(a, x) \in(0,1] \times\left[0, \tilde{x}_{0}\right]$. So, by $(2.4)$ and dominated convergence,

$$
p d_{\mathrm{Ko}}(p) \geq p\left[F_{\infty}\left(\tilde{x}_{0}\right)-F_{p}\left(\tilde{x}_{0}\right)\right]=-\int_{0}^{\tilde{x}_{0}} \frac{f_{1 / a}(x)-f_{\infty}(x)}{a} \mathrm{~d} x \underset{a \downarrow 0}{\longrightarrow}-\int_{0}^{\tilde{x}_{0}} \lambda(x) \mathrm{d} x=\frac{\left(\tilde{x}_{0}^{3}+\tilde{x}_{0}\right) \varphi\left(\tilde{x}_{0}\right)}{4}=C,
$$

where $\lambda(x)$ is defined in (2.4). This, together with (1.6), implies (1.8). The proof of Theorem 1.2 is now complete.

\section{REFERENCES}

[1] S. Bourguin and C. Tudor, Malliavin calculus and self normalized sums. Seminaire de Propabilités XLV. Lect. Notes Math. Springer (2013) 323.

[2] T. Cacoullos, V. Papathanasiou, and S.A. Utev, Variational inequalities with examples and an application to the central limit theorem. Ann. Probab. 22 (1994) 1607-1618.

[3] I. Pinelis, Monotone tail and moment ratio properties of Student's family of distributions. Version 2. http://arxiv.org/abs/ 1101.3289 (2012).

[4] I. Pinelis, On the Berry-Esseen bound for the Student statistic. Version 2. http://arxiv.org/abs/1101.3286 (2012).

[5] I. Pinelis, Exact bounds on the closeness between the Student and standard normal distributions. Version 2. http://arxiv . org/abs/1101.3328 (2013).

[6] I. Pinelis and R. Molzon, Berry-Esséen bounds for general nonlinear statistics, with applications to Pearson's and non-central Student's and Hotelling's. http://arxiv.org/abs/math/0701806 (2009).

[7] R.S. Pinkham and M.B. Wilk, Tail areas of the $t$-distribution from a Mills'-ratio-like expansion. Ann. Math. Statist. 34 (1963) $335-337$.

[8] H. Scheffé, A useful convergence theorem for probability distributions. Ann. Math. Statist. 18 (1947) $434-438$.

[9] R. Shimizu, Error bounds for asymptotic expansion of the scale mixtures of the normal distribution. Ann. Inst. Statist. Math. 39 (1987) 611-622. 